\title{
COMPLEX ANALYTIC MAPPINGS ${ }^{1}$
}

\section{LEO SARIO}

1. Introduction. The starting point of the value distribution theory of complex analytic mappings was Picard's classical theorem: an analytic mapping of the complex plane into the extended plane can omit at most two points. During the 83 years that have elapsed since the publication of Picard's theorem, the evolution has taken place towards greater generality: the Picard-Nevanlinna theory was first extended from the plane to more general plane regions by af Hällström [4], and then to various Riemann surfaces by Ahlfors $[1 ; 2]$, Heins [5], Kunugui [7], Kuramochi [8], Myrberg [9], Noshiro [10], Ohtsuka [11], Parreau [12], Tamura [16], Tsuji [17], Tumura [18], and others.

The most general result was obtained in 1960 by Chern [3], who considered as domain $R$ a closed Riemann surface less a finite number of points, and as range $S$ a closed Riemann surface. He showed that under a complex analytic mapping $\zeta=f(z)$ of $R$ into $S, z$ and $\zeta$ being the local complex parameters, the number $P$ of Picard points, and more generally the defect sum, cannot exceed the negative of the Euler characteristic of $S$ :

$$
P \leqq-e_{S} .
$$

This beautiful result of Chern's paves the way to the following question: can generality be pushed further by allowing both $R$ and $S$ to be arbitrary? A priori this did not seem likely. In fact, Heins [5] had exhibited a rather simple Riemann surface of infinite genus, which carried meromorphic functions with infinitely many Picard values. A closer look at the situation reveals, however, rather interesting new aspects. To this end let us consider current methods and see if we can introduce simplifications which permit greater generality. Our report has appeared in extenso in [14].

An address delivered before the Berkeley meeting of the Society on January 25, 1963 by invitation of the Committee to Select Hour Speakers for Far Western Sectional Meetings; received by the editors February 1, 1963.

1 The work was sponsored by the Army Research Office (Durham), Grant DAARO(D)-31-124-G40, University of California, Los Angeles. 
2. Proximity function. The first tool needed is a function to describe the proximity of a generic point $\zeta \in S$ to a given point $a \in S$. The standard method is the following: one first forms a conformal metric with area element $d \omega=\lambda^{2} d S$, where $d S$ is Euclidean area element in the parametric disk, and $\lambda$ is covariant and strictly positive. Throughout our presentation let $t(\zeta, a, b)$ be a harmonic function of the variable $\zeta$ on $S$ with a positive logarithmic pole at $a$ and a negative logarithmic pole at $b$. One integrates $t$ with respect to $d \omega(b)$ over $S$. The resulting function

$$
q(\zeta, a)=\int_{S} t(\zeta, a, b) d \omega(b)
$$

is bounded below and has a positive logarithmic singularity at $a$. It thus qualifies to describe the proximity of $\zeta$ to $a$. Moreover, $\Delta q$ is simply the "density" of the metric:

$$
\Delta q=\lambda^{2} .
$$

This makes it possible to use effectively the standard relations between line and area integrals.

There are, however, two drawbacks to this approach. First, if $S$ is open, it seems difficult, if not impossible, to establish the convergence of integral (2). Second, even when $S$ is closed, a rather lengthy reasoning in partial differential equations is needed to show that $\Delta q$ actually is $\lambda^{2}$. If $S$ is open, there seems to be no way of putting the reasoning through.

To overcome this difficulty, I suggest the following reversal of the process: start with a function

$$
t_{0}(\zeta)=t\left(\zeta, \zeta_{0}, \zeta_{1}\right)
$$

with given $\zeta_{0}, \zeta_{1} \in S$. The singularities, together with a normalization of the additive constant, uniquely determine $t_{0}$ if $S$ is closed. If $S$ is bordered and compact, then we add the condition that the normal derivative vanish on the border. If $S$ is open, we take for $t_{0}$ the directed limit of the function thus constructed on a bordered subregion as the subregion exhausts $S$. The limiting function is a special case of the socalled principal function, and its existence is assured by the related linear operator method [15]. The function

$$
s_{0}(\zeta)=\log \left(1+e^{t_{0}(\zeta)}\right)
$$

is bounded below but continues to have a positive logarithmic pole at $\zeta_{0}$. For any other point $a$ take $t\left(\zeta, a, \zeta_{0}\right)$ and add it to $s_{0}(\zeta)$. The singularities at $\zeta_{0}$ cancel and the function 


$$
s(\zeta, a)=s_{0}(\zeta)+t\left(\zeta, a, \zeta_{0}\right)
$$

is bounded below and has a positive logarithmic singularity at $a$. We choose this function to describe the proximity of $\zeta$ to $a$ : closer proximity gives greater values. The function exists on every Riemann surface $S$, open or closed, of finite or infinite genus.

Having formed $s$, we now introduce a conformal metric with area element $\lambda^{2} d S$ by choosing the density $\lambda^{2}=\Delta s=\Delta s_{0}$. It is independent of $a$. Thus the convergence problem of (2) and the proof of (3) are eliminated, and the metric is obtained on an arbitrary $S$.

The metric has zeroes of $\lambda$ at the zeroes of grad $t_{0}$. But these zeroes turn out to be helpful, and, in fact, constitute a rather essential aspect of the theory.

In passing, we remark that the Gaussian curvature of our metric is constantly 1 , and its total area $\int_{S} d \omega=2 \pi$. As a by-product we thus have, on an arbitrary Riemann surface, a conformal metric (with zeroes of $\lambda$ ) of constant curvature and finite total area.

3. The characteristic function. We can now at once write down the first main theorem. It was earlier considered from different viewpoints by Heins [6], Kuramochi [8], Myrberg [9], and Parreau [12]. Here we give it in a form that directly serves the sole purpose of our report: the second main theorem and Picard's theorem on an arbitrary $R$.

Remove from $R$ a parametric disk $R_{0}$ with boundary $\beta_{0}$, and consider an adjacent relatively compact subregion $\Omega$ with boundary $\beta_{0} \cup \beta_{\Omega}$. On $\Omega$ form the harmonic function $u$ with $u=0$ on $\beta_{0}, u=k(\Omega)$, a constant, on $\beta_{\Omega}$, such that the flux $\int_{\beta_{0}} d u^{*}=1$. For $h \in[0, k]$ consider the level line $\beta_{h}=u^{-1}(h)$ and the region $\Omega_{h}=u^{-1}((0, h))$ between $\beta_{0}$ and $\beta_{h}$. Given a point $a \in S$ let $z_{j}$ be its inverse images under $t$ and denote their number in $\Omega_{h}$ by $\nu(h, a)$.

For the counting function we take

$$
A(h, a)=2 \pi \int_{0}^{h} \nu(h, a) d h .
$$

It reflects the frequency of $a$-points of $f$ on $R$. In particular, it vanishes for a Picard point $a$. We choose the proximity function

$$
B(h, a)=\int_{\beta_{h}} s(f(z), a) d u^{*} .
$$

Its geometric meaning is clear: it is the mean proximity to $a$ of the image $f\left(\beta_{h}\right)$ under $f$ of $\beta_{h}$. Finally, for the characteristic function we take 


$$
C(h)=\int_{\Omega_{h}}(h-u(z)) d \omega(f(z)) .
$$

In contrast with the classical theory, the integrand here depends on the region; but this will cause no difficulty. The geometric meaning continues to be that $C^{\prime}(h)$ is the area $\int_{\Omega_{h}} d \omega(f(z))$ of the multi-sheeted image under $f$ of $\Omega_{h}$ over $S$.

A simple application of Stokes' formula to $\Omega_{h}$, from which we first remove small disks about the $z_{j}$ and let them shrink to their centers, gives the

FiRST MAIN THEOREM. For every regular region $\Omega \subset R$ under an analytic mapping of an arbitrary Riemann surface $R$ into another arbitrary Riemann surface $S$,

$$
A(k, a)+B(k, a)=C(k)+O(k) .
$$

It turns out that for all functions of interest $C$ grows more rapidly than $k$. Thus $O(k)$ is negligible, and the elegant classical balance prevails: the $(A+B)$-affinity, so to speak, of $f$ is the same for all points $a \in S$. In particular, for a Picard point $a, A \equiv 0$ and we have a strong proximity of $f\left(\beta_{h}\right)$ to $a$.

4. Picard points. We now come to the main question: how many Picard points $a_{1}, \cdots, a_{q}$ can exist? The answer is given by the second main theorem which we shall give for mappings of an arbitrary $R$ into a closed $S$. The reasoning remains valid mutatis mutandis for an arbitrary $S$.

It is well known that in the classical second main theorem the remainder cannot be estimated for all values of the variable $r$. It is the integral of the integral of the remainder that can be given a dominating function. The remainder itself can behave arbitrarily wildly in certain intervals whose length can be estimated but which must be omitted in stating the second main theorem. When one then takes the defect relation, these exceptional intervals and the related changing of the coordinate system with varying $\Omega$ prevent the use of directed limits. But ordinary limits cannot be employed on an arbitrary Riemann surface $R$ : there is no one single parameter that would give an exhaustion of $R$. Thus the classical theory does not carry over to the general case.

This difficulty can be overcome by the following simple device. We replace the proximity function $B$ by the integral of its integral. Geometrically the first integration means that we replace the mean proximity of the image curve $f\left(\beta_{h}\right)$ by what is just as natural if not more so, the mean proximity of the image region $f\left(\Omega_{h}\right)$, and then we take 
the integral of this. Analytically this means that, in some sense, we bring all quantities involved to the same level of integration. Then the remainder term in the second main theorem has an estimate for every subregion $\Omega$, directed limits can be employed, and the theory established on an arbitrary $R$.

The actual derivation of the second main theorem consists of little more than another application of Stokes' formula. The proof is further facilitated by the presence of the zeroes of $\lambda$ we referred to earlier. Their number is the Euler characteristic of the punctured (at $\zeta_{0}$ and $\left.\zeta_{1}\right) S$ and we obtain $e_{S}$ without using the Gauss-Bonnet formula. Geometrically, in the fiber bundle of unit tangent vectors on $S$, we do not integrate over a cross-section but over a base domain on $S$. This makes it unnecessary to set up the fiber bundle, and we can dispense with borrowing from differential geometry.

When the computations are carried out, we obtain the following

SeCOND MAIN THEOREM. For any $\Omega \subset R$ under an analytic mapping of an arbitrary $R$ into a closed $S$,

$$
\begin{aligned}
\left(q+e_{S}\right) C_{2}(k)< & \sum_{i=1}^{q} A_{2}\left(k, a_{i}\right)-A_{2}\left(k, f^{\prime}\right)+E_{2}(k) \\
& +O\left(k^{3}+k^{2} \log C(k)\right),
\end{aligned}
$$

where $C_{2}(k)$ is the integral of the integral from 0 to $k$ of $C(h), A_{2}\left(k, f^{\prime}\right)$ counts the multiple-points of $f(\Omega)$, and $E_{2}(k)$ is the $(2 \pi-f o l d)$ thrice integrated Euler characteristic e $(h)$ of $\Omega_{h}$.

The remainder term $O$ is negligible for the nondegenerate class of functions which was given the following elegant characterization by my student Rao: there must exist a constant $0<\alpha<1$ such that $k / C(k) \rightarrow 0$ and, for $\Omega_{0}=\bar{R}_{0} \cup \Omega$,

$$
\lim _{\Omega_{0} \rightarrow R} \frac{\log C(k)}{C(\alpha k)}=0 .
$$

For these functions we can now introduce the defect

$$
\delta(a)=1-\limsup _{\Omega_{0} \rightarrow R} \frac{A_{2}(k, a)}{C_{2}(k)} .
$$

For a Picard point $a$, this defect is obviously $=1$. We also introduce the ramification index

$$
\theta=\liminf _{\Omega_{0} \rightarrow R} \frac{A_{2}(k, f)}{C_{2}(k)}
$$


and what could be called the Euler index

$$
\eta=\lim _{\Omega_{0} \rightarrow R} \frac{E_{2}(k)}{C_{2}(k)} .
$$

We obtain at once:

DefECT RELATION. For nondegenerate analytic mappings of an arbitrary Riemann surface $R$ into a closed Riemann surface $S$,

$$
\sum \delta(a)+\theta \leqq \eta-e_{S} .
$$

We can now throw some light into the Heins phenomenon. If $C_{2}$ grows less rapidly than $E_{2}$, then $\eta=\infty$ and there can be infinitely many Picard points. But even in the elementary case of the disk $R$, e.g., the identity mapping omits infinitely many points, in fact the entire complement of the disk. The problem of Picard values becomes interesting only if a growth condition is imposed upon the characteristic function. For the disk such a condition is well known. For an arbitrary Riemann surface we now have, in addition to the nondegeneracy conditions $k / C(k) \rightarrow 0$ and $\log C(k) / C(\alpha k) \rightarrow 0$, the essential condition reflecting the topology of $R$ : the characteristic function must grow at least as rapidly as the Euler characteristic. For these mappings we have what we set out to find, a Picard theorem on an arbitrary $R$ : the number of Picard values cannot exceed the excess of $\eta$ over $e_{S}$.

$$
P \leqq \eta-e_{S} .
$$

In the case of a sphere $S$, i.e., for meromorphic functions on arbitrary Riemann surfaces, the bound $2+\eta$ was shown to be sharp by an interesting example constructed by my student Rodin [13]. In the classical case of meromorphic functions in the plane, we have an elementary proof of the defect relation, and a second main theorem without exceptional intervals.

\section{REFERENCES}

1. L. Ahlfors, Zur Theorie der Überlagerungsfiächen, Acta Math. 65 (1935), 157194.

2. - Über die Anwendung differentialgeometrischer Methoden zur Untersuchung der Überlagerungsflächen, Ann. Acad. Sci. Fenn. Ser. A II 6 (1937).

3. S. Chern, Complex analytic mappings of Riemann surfaces. I, Amer. J. Math. 82 (1960), 323-337.

4. G. af Hällström, Über meromorphe Funktionen mit mehrfach zusammenhangenden Existenzgebieten, Acta Acad. Åbo. Math. Phys. 12 (1940), no. 8. 317.

5. M. Heins, Riemann surfaces of infinite genus, Ann. of Math. (2) 55 (1952), 296-

6. - Lindelöfian maps, Ann. of Math. (2) 62 (1955), 418-446. 
7. K. Kunugui, Sur l'allure d'une fonction analytique uniforme au voisinage d'un point frontière de son domaine de definition, Japan. J. Math. 18 (1942), 1-39.

8. Z. Kuramochi, On covering surfaces, Osaka Math. J. 5 (1953), 155-201.

9. L. Myrberg, Über meromorphe Funktionen und Kovarianten auf Riemannschen Flächen, Ann. Acad. Sci. Fenn. Ser. A I 244 (1957).

10. K. Noshiro, Open Riemann surfaces with null boundary, Nagoya Math. J. 3 (1951), 73-79.

11. M. Ohtsuka, Reading of the paper On covering surfaces by Z. Kuramochi, Chapter I (mimeograph).

12. M. Parreau, Sur les moyennes des fonctions harmoniques et analytiques et la classification des surfaces de Riemann, Ann. Inst. Fourier (Grenoble) 3 (1951), 103-197.

13. B. Rodin, Reproducing formulas on Riemann surfaces, Doctoral Dissertation, Univ. of California, Los Angeles, Calif., 1961.

14. L. Sario, Value distribution under analytic mappings of arbitrary Riemann surfaces, Acta Math. 109 (1963), 1-10.

15. - A linear operator method on arbitrary Riemann surfaces, Trans. Amer. Math. Soc. 72 (1952), 281-295.

16. J. Tamura, Meromorphic functions on open Riemann surfaces, Sci. Papers Coll. Gen. Ed. Univ. Tokyo 9 (1959), 175-186.

17. M. Tsuji, Theory of meromorphic functions on an open Riemann surface with null boundary, Nagoya Math. J. 6 (1953), 137-150.

18. Y. Tumura, Quelques applications de la theorie de M. Ahlfors, Japan. J. Math. 18 (1942), 303-322.

University of California, Los Angeles 